\title{
MODELAGEM DO FENÔMENO DE TRANSFERÊNCIA DE MASSA NA HIDRATAÇÃO DO CEREAL MATINAL
}

Modeling the mass transfer phenomenon in the hydration of morning cereal

Modelado del fenómeno de transferencia de masa en la hidratación del cereal matinal

\section{Andréia Ibiapina ${ }^{* 1}$, Eduardo Henrique Silva de Oliveira ${ }^{1}$, Glêndara Aparecida de Souza Martins ${ }^{1}$, Warley Gramacho da Silva ${ }^{1}$.}

${ }^{1}$ Laboratório de Cinética e Modelagem de Processos, Engenharia de Alimentos, Universidade Federal do Tocantins, Palmas, Tocantins, Brasil.

*Correspondência: Laboratório de Cinética e Modelagem de Processos, Universidade Federal do Tocantins, Av. NS 15, ALCNO 14 - Bloco II, Sala 06, 109 Norte, Palmas, Tocantins, Brasil. CEP:77.010-090. e-mail ibiapinaandreia@gmail.com.

Artigo recebido em 29/04/2019 aprovado em 03/05/2019 publicado em 16/06/2019.

\section{RESUMO}

A crocância do cereal matinal está associada a qualidade do produto e é de suma importância dentre suas características sensoriais. O processo de hidratação o qual ele geralmente sofre antes de ser ingerido, pode ocasionar alterações nesta característica e alguns fatores como, quantidade de fluido disponível, temperatura e tempo de hidratação afetam diretamente esse processo. O objetivo do trabalho foi utilizar modelos empíricos (Cinético de $1^{\text {a }}$ Ordem e Exponencial) afim de avaliar os parâmetros de hidratação de um cereal matinal hidratado com leite. A hidratação ocorreu em 3 proporções, 3 temperaturas e durante 2 horas, sendo analisadas amostras desse cereal e do leite a cada 15 minutos de hidratação. Dos modelos empíricos, o modelo exponencial foi o que melhor descreveu a cinética de hidratação, porém, nenhum dos dois modelos utilizados mostraram parâmetros de ajustes tão satisfatórios, fazendo necessário o uso de outro modelo que melhor se ajuste aos dados experimentais.

Palavras-chave: Absorção; Cinética de hidratação, Cereal matinal.

\section{ABSTRACT}

The crunchiness of the morning cereal is associated with product quality and is of paramount importance among its sensorial characteristics. The process of hydration which it usually suffers before being ingested may cause changes in this characteristic and some factors such as amount of available fluid, temperature and time of hydration directly affect this process. The objective of the study was to use empirical models (1st Order Kinetic and Exponential) in order to evaluate the hydration parameters of a milk cereal hydrated with milk. The hydration occurred in 3 proportions, 3 temperatures and during 2 hours, being analyzed samples of this cereal and the milk every 15 minutes of hydration. From the empirical models, the exponential model was the one that best described the hydration kinetics. However, none of the two models showed satisfactory adjustment parameters, making it necessary to use another model that best fits the experimental data.

Keywords: Absorption; Hydration kinetics; Breakfast cereal.

\section{RESUMEN}

La crocancia del cereal matinal está asociada a la calidad del producto y es de suma importancia entre sus características sensoriales. El proceso de hidratación que generalmente sufre antes de ser ingerido, puede ocasionar cambios en esta característica y algunos factores como, cantidad de fluido disponible, temperatura y tiempo de hidratación afectan directamente ese proceso. El objetivo del trabajo fue utilizar modelos empíricos (Cinético de $1^{a}$ Orden y Exponencial) a fin de evaluar los parámetros de hidratación de un cereal matinal hidratado con leche. La hidratación ocurrió en 3 proporciones, 3 temperaturas y durante 2 horas, siendo analizadas muestras de ese cereal y de la leche cada 15 minutos de hidratación. De los modelos empíricos, el modelo exponencial fue el que mejor 
describió la cinética de hidratación, sin embargo, ninguno de los dos modelos utilizados mostraron parámetros de ajustes tan satisfactorios, haciendo necesario el uso de otro modelo que mejor se ajuste a los datos experimentales. Descriptores: absorción; Cinética de hidratación, Cereal matinal.

\section{INTRODUÇÃO}

Os alimentos derivados de cereais são extremamente eficazes para a ingestão de calorias da população de todas as faixas etárias, além de seu uso como matéria prima ser frequente na indústria alimentícia (SINGH et al., 2014). Pensando em alimentos de fácil preparo direcionados ao pouco tempo do dia-a-dia, foram desenvolvidos os cereais matinais, que são produtos extrusados com alto teor de proteína, carboidratos e fibras, fabricados a base de milho e consumidos, em sua maioria, através da hidratação com leite. Por ser um alimento muito nutritivo é considerado um alimento ideal para uma refeição matinal (MIKALOUSKI et al., 2014).

O leite, do ponto de vista nutricional é um dos alimentos mais completos, possuindo alto teor de proteínas (caseína e albumina) e sais minerais, além de ser grande fonte de cálcio possui alta composição de água e alguns carboidratos, como a Lactose, gorduras e vitaminas (MACHADO et al., 2014).

$\mathrm{O}$ aumento da população intolerante a lactose $\left(\mathrm{C}_{12} \mathrm{H}_{22} \mathrm{O}_{11}\right)$, ou seja, de indivíduos os quais o organismo possui dificuldades de hidrolisar esse açúcar presente no leite, é evidente e preocupante (MATTAR et al., 2010). No intuito de melhorar a qualidade de vida desse grupo nutricional, possibilitando aos indivíduos acesso ao leite sem prejudicar sua saúde, a indústria desenvolveu o leite sem a presença da lactose (FAEDO et al., 2013)

De acordo com Faedo et al., (2013), o setor lácteo é de suma importância para a indústria brasileira, sendo que, apesar do grande aumento de pessoas alérgicas à proteína do leite e à lactose, várias medidas vêm sendo tomadas para promover o acesso a esse produto sem danos à saúde do consumidor, tais como os processos de hidrólise da lactose que consiste na quebra desse carboidrato, tornando o leite próprio para o consumo dos indivíduos intolerantes.

Muitos estudos relacionados com a hidratação de grãos como soja, feijão, arroz parbolizado e cereais vem sendo feitos para a análise da cinética de adsorção e absorção de fluido para avaliar a influência que a temperatura exerce sobre a taxa de hidratação. Os modelos matemáticos são ferramentas importantes na concepção e otimização de processos de desidratação e hidratação. Os quais permitem predizer e simular o comportamento de determinados parâmetros e processos, podendo ser utilizado modelos empíricos e fenomenológicos (ANSARI et al, 2015; BALBINOTI et al., 2018; LISBOA et al., 2015).

Com isso, o principal objetivo deste trabalho é realizar a modelagem matemática do fenômeno de transferência de massa no processo de hidratação do cereal matinal com leite desnatado sem lactose, utilizando os modelos de Exponencial e o Cinético de $1^{\text {a }}$ Ordem.

\section{MATERIAIS E MÉTODOS}

\section{Hidratação do cereal matinal}

O cereal matinal utilizado foi o de milho (sem açúcar), e o liquido de imersão foi o leite UHT Desnatado, ambos adquiridos em comércio local da cidade Palmas - TO, na sua forma pronta para consumo. $\mathrm{O}$ experimento consistiu em um fatorial $3 \times 3 \times 8$, onde os fatores analisados na hidratação foram: proporção leite/cereal, tempo de hidratação e a temperatura de imersão. Os cereais foram imersos no leite em proporções de 1:10, 1:12,5 e 1:15. As temperaturas de ensaio foram 45,50 e $55^{\circ} \mathrm{C}$, e a 
hidratação ocorreu durante duas horas, com retirada do cereal a cada 15 minutos $(15,30,45,60,75,90,105$ e 120 minutos) para pesagem e análise da cinética de absorção do leite, onde o excesso de leite foi retirado com o auxílio de papel filtro antes da pesagem. A hidratação foi feita em triplicata, com 2 repetições.

\section{Modelagem matemática da absorção do cereal matinal}

Para avaliação dos resultados, foram utilizados dois modelos matemáticos empíricos, sendo eles os modelos exponencial, representado pela equação 1 no qual a k2 é a constante do modelo (COX et al., 2012), e o modelo Cinético de $1^{\text {a }}$ Ordem, onde a constante k1 é a taxa de hidratação, representado pela equação 2 (KROKIDA e MARINOS, 2003), para avaliar se o processo de umidificação exercia alguma influência sobre o cereal. Os resultados foram analisados utilizando o software Statistica 7.

$$
\begin{gathered}
U_{t}=U_{e q}\left(1-\exp \left(-k_{2} t\right)\right) \\
\frac{U_{t}-U_{e q}}{U_{0}-U_{e q}}=\exp \left(-k_{1} t\right)
\end{gathered}
$$

\section{RESULTADOS E DISCUSSÃO}

O modelo cinético de $1^{\text {a }}$ ordem é baseado no modelo de difusão da segunda lei de Fick para diferentes geometrias onde a constante do modelo, $\mathrm{k}_{1}$, é a taxa de hidratação (KROKIDA e MARINOS, 2003). Verifica-se, na tabela 1 , que quanto maior a temperatura, maior o valor de $\mathrm{k}_{1} \mathrm{e}$, consequentemente, maior a taxa de hidratação do cereal. O modelo cinético de $1^{\text {a }}$ ordem é um modelo de três parâmetros, associado à umidade em um dado instante, umidade de saturação e umidade inicial (VOLPE et al., 2015).

No modelo exponencial, a constante $\mathrm{k}_{2}$ está relacionada com a velocidade de absorção. Na Tabela 1, estão descritos os valores de $\mathrm{k}_{2}$ durante a absorção. É possível observar que em temperaturas mais elevadas a velocidade de absorção é maior. Resultados semelhantes são reportados pela literatura para hidratação de grãos de milho transgênico, onde os autores observaram rápida absorção de água pelos grãos e posterior redução desse fenômeno ao aproximar-se de condições de equilíbrio (MARQUES et al., 2015).

Tabela 1: Parâmetros dos modelos de $1^{\mathrm{a}}$ Ordem e Exponencial aplicados à cinética de absorção de umidade em cereal matinal.

\begin{tabular}{cccccc}
\hline & & \multicolumn{2}{c}{$\mathbf{1}^{\mathbf{a}}$ Ordem } & \multicolumn{2}{c}{ Exponencial } \\
\hline Proporção & $\mathbf{T}\left({ }^{\mathbf{0}} \mathbf{C}\right)$ & $\mathbf{K}_{\mathbf{1}}$ & $\mathbf{U e q}$ & $\mathbf{k}_{\mathbf{2}}$ & $\mathbf{U e q}$ \\
\hline \multirow{3}{*}{$\mathbf{1 / 1 0}$} & 45 & 0,08 & 20,83 & 22,69422 & 20,46075 \\
& 50 & 0,09 & 22,95 & 25,02503 & 21,70000 \\
& 55 & 0,10 & 25,23 & 25,08771 & 24,21150 \\
\hline \multirow{3}{*}{$\mathbf{1 / 1 2 , 5}$} & 45 & 0,10 & 20,19 & 23,62215 & 18,93900 \\
& 50 & 0,13 & 21,79 & 21,76316 & 20,89787 \\
& 55 & 0,12 & 26,13 & 27,34224 & 22,82150 \\
\hline \multirow{3}{*}{$\mathbf{1 / 1 5}$} & 45 & 0,10 & 20,68 & 21,77156 & 19,10637 \\
& 50 & 0,14 & 23,61 & 26,73078 & 20,67088 \\
& 55 & 0,12 & 27,35 & 29,44897 & 21,70762 \\
\hline
\end{tabular}

Os valores dos coeficientes de determinação $\left(\mathrm{R}^{2}\right)$, erro médio relativo (SE) e erro padrão da estimativa (P) para os modelos de $1^{\text {a }}$ ordem $\mathrm{e}$ Exponencial, ajustados durante a hidratação do cereal, 
estão apresentados na Tabela 1. Quanto mais próximo de $100 \%$ for o coeficiente de determinação e menor o erro médio relativo, melhor o ajuste do modelo. Sendo assim, o modelo de Exponencial apresentou os melhores resultados em comparação ao modelo de $1^{\mathrm{a}}$ ordem, uma vez que o coeficiente de determinação do modelo Exponencial chegou a 93\%, porém, quando se observa os valores de erro médio e erro relativo, nenhum dos dois modelos apresentam valores de ajuste satisfatório. Sendo assim, o modelo que mais se ajustou a hidratação de cereal matinal com leite, dentre os estudados, foi o modelo Exponencial. Este comportamento também pode ser observado na Figura
1 que contém a correspondência entre os valores experimentais e estimados para os modelos. A combinação dos valores do coeficiente de determinação, erro médio relativo e erro padrão da estimativa satisfatórios demonstra o melhor modelo de ajuste (PAIXÃO et al., 2017).

Tabela 2: Índices estatísticos para modelagem da hidratação do cereal com leite desnatado

\begin{tabular}{cccccccc}
\hline & \multicolumn{3}{c}{$\mathbf{1}^{\mathbf{a}}$ Ordem } & \multicolumn{3}{c}{ Exponencial } \\
\hline Proporção & $\mathbf{T}^{\mathbf{0}} \mathbf{C}$ & $\mathbf{R}^{\mathbf{2}}$ & $\mathbf{S E}$ & $\mathbf{P}(\boldsymbol{\%})$ & $\mathbf{R}^{\mathbf{2}}$ & $\mathbf{S E}$ & $\mathbf{P}(\boldsymbol{\%})$ \\
& 45 & 0,83 & 7,61 & 14,14 & 0,90 & 2,50 & 15,82 \\
$\mathbf{P 1}$ & 50 & 0,74 & 7,54 & 21,61 & 0,93 & 2,14 & 13,73 \\
& 55 & 0,86 & 6,47 & 10,45 & 0,92 & 2,81 & 17,29 \\
\hline \multirow{2}{*}{$\mathbf{P 2}$} & 45 & 0,90 & 15,77 & 21,27 & 0,89 & 2,32 & 16,54 \\
& 50 & 0,81 & 5,02 & 11,33 & 0,90 & 2,52 & 16,29 \\
& 55 & 0,72 & 3,05 & 20,47 & 0,92 & 2,51 & 16,23 \\
\hline \multirow{3}{*}{$\mathbf{P 3}$} & 45 & 0,69 & 16,83 & 11,22 & 0,90 & 2,26 & 15,83 \\
& 50 & 0,89 & 10,06 & 16,66 & 0,85 & 15,06 & 20,60 \\
& 55 & 0,87 & 6,54 & 17,81 & 0,88 & 3,07 & 19,74
\end{tabular}

Na Figura 1 estão apresentados os gráficos de correspondência entre os teores de leite experimentais e estimados pelos modelos de $1^{\text {a }}$ Ordem e Exponencial nas proporções de 1/10 (Figura 1a), 1/12,5 (Figura 1b) e 1/15 (Figura 1c), com seus coeficientes variando em função das temperaturas $\left(45 ; 50\right.$ e $\left.55^{\circ} \mathrm{C}\right)$ e proporções estudadas $(1 / 10 ; 1 / 12,5$ e $1 / 15)$, bem como os valores de erro padrão da estimativa. O modelo exponencial foi o que melhor se ajustou ao processo, como já citado acima, por causa de seus parâmetros mais satisfatórios apresentados na Tabela 2. O modelo exponencial mostrou melhores ajustes quando comparado a outros modelos no estudo da capacidade de absorção de sementes de lentilha em diferentes temperaturas (OROIAN, 2017) e no processo de hidratação de chips de mandioca mostrando uma maior absorção nos primeiros momentos de hidratação (AJALA et al., 2015). 
Figura 1: Correspondência entre os valores experimentais e estimados para os modelos de $1^{\mathrm{a}}$ ordem e Exponencial, em diferentes temperaturas, durante a hidratação do cereal matinal com leite desnatado e hidrolisado, para (a) proporção de 1/10, (b) proporção 1/12,5 e (c) proporção 1/15.
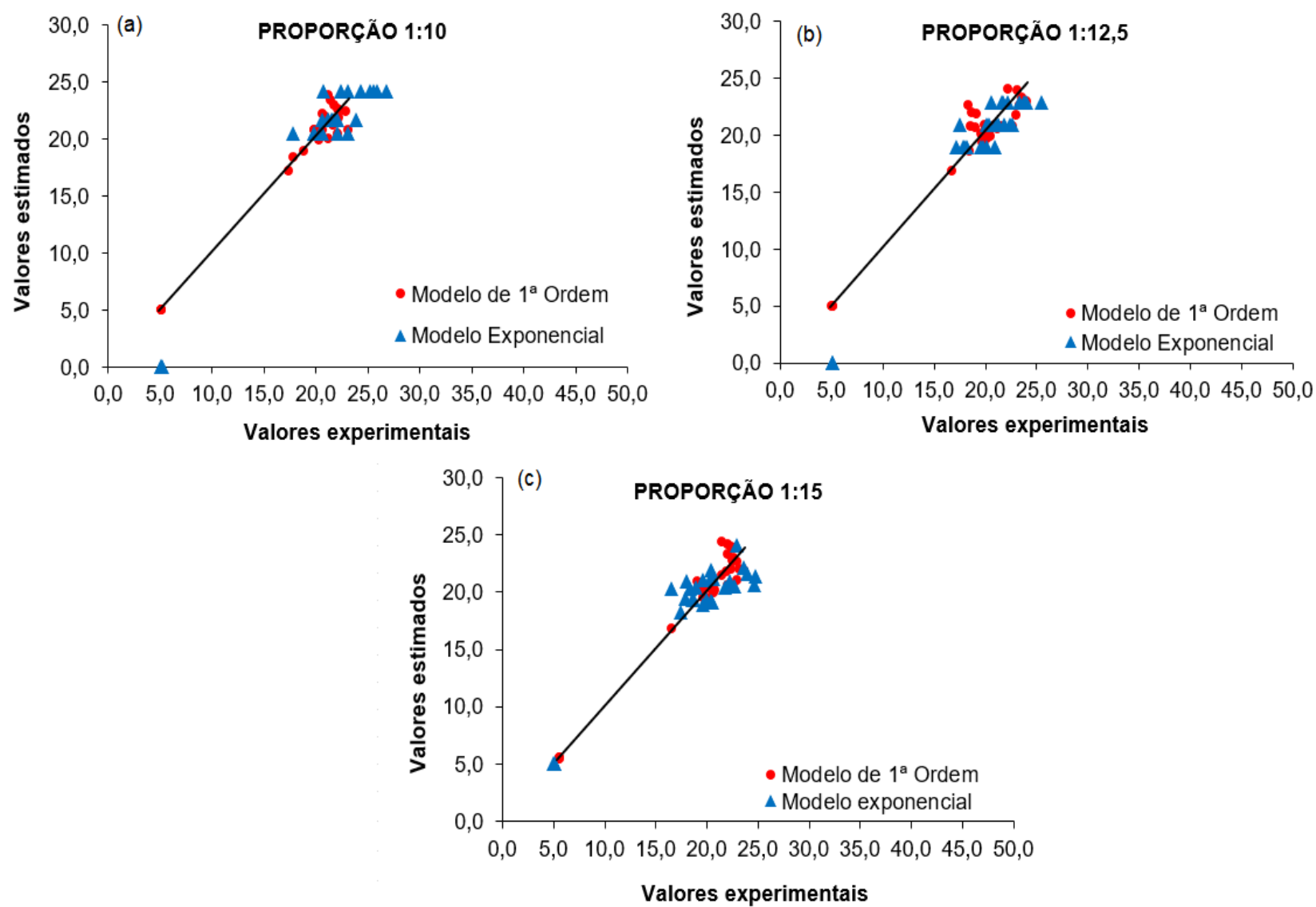

\section{CONCLUSÃO}

Dentre os modelos matemáticos empíricos utilizados o que melhor se ajustou aos dados experimentais foi o modelo Exponencial, uma vez que, apresentou valores de coeficiente de determinação maiores e valores de Erro médio relativo e Erro padrão da estimativa menores quando comparados aos encontrados pelo modelo cinético de $1^{\mathrm{a}}$ ordem.

\section{AGRADECIMENTO}

Agradecemos à Universidade Federal do Tocantins e ao CNPQ pela ajuda financeira para realização do projeto e por ceder espaço para sua execução.

Todos os autores declararam não haver qualquer potencial conflito de interesses referente a este artigo.

\section{REFERÊNCIAS}

AJAlA, A. S., AJAlA, F. A., OYEDELE, J. O. Rehydration characteristics and modeling of cassava chips. Am. J. Eng. Res, 4, 45-49. 2015.

ANSARI, S., HOSSEINI, E., ASADI, G. H., MAFTOONAZAD, N., FARAHNAKY, A. Modeling the Rehydration Behavior of Dried Figs. Journal of Agricultural Science and Technology. $v$. 17, p. 133-144. 2015.

BALBINOTI, T. C. V., JORGE, L. M. M., JORGE, R. M. M. Mathematical modeling of paddy (Oryza sativa) hydration in different thermal conditions assisted by Raman spectroscopy. Journal of Cereal Science, 79, 390-398. 2018.

COX, S., GUPTA, S., ABUGHANNAM, N. Effect of Different Rehydration Temperatures on the Moisture, Content of Phenolic Compounds, Antioxidant Capacity and Textural Properties of Edible Irish Brown Seaweed. LWT - Food Science and Technology, v. 47, p. 300-307. 2012.

FAEDO, R.; BRIÃO, V. B.; CASTOLDI, S.; GIRARDELLI, L.; MILANI, A. Obtenção de leite com baixo teor de lactose por processos de separação 
por membranas associados à hidrólise enzimática. Revista CIATEC-UPF, v. 5, n. 1, p. 4454, 2013.

KROKIDA, M. K.; MARINOS-KOURIS, D. Rehydration Kinetics of Dehydrated Products. J. Food Eng., 57: 1-7. 2003.

LISBOA, J. F., SILVA, J. N., CSAVALCANTI, M. T., SILVA, E. M., GONÇALVES, M. C. Análise da hidratação de grãos de alpiste. Revista Brasileira de Engenharia Agricola e Ambiental-Agriambi, v. 19, n. 3. 2015.

MACHADO, A. R. T., CAMPOS, J. E. C., CLARETO, S. S., MORAES, A. L. L. Características físico-químicas e sensoriais de três marcas de leite de vaca pasteurizado e comercializado na cidade de Alfenas-MG. Revista da Universidade Vale do Rio Verde. v. 12, n. 2, p. 93-99. 2014.

MARQUES, B., JORGE, L., JORGE, R. Cinética de hidratação e da liberação de sólidos solúveis do milho geneticamente modificado e de sua isolinha. Blucher Chemical Engineering Proceedings, 1(2), 29582965. 2015.

MATTAR, R.; MAZZO, D. F. C. Intolerância à lactose: mudança de paradigmas com a biologia molecular. Revista da Associação Médica Brasileira, v. 56, n. 2, p. 230-236, 2010.

MIKALOUSKI, F. B. S., MONTEIRO, A. R. G., MARQUES, D. R., MONTEIRO, C. C. F., BENOSSI, L. Influência da granulometria da matéria-prima na expansão de extrusados de milho/Influence of raw material particle size on the expansion of extruded corn. Brazilian Journal of Food Technology, 17(1), 28. 2014.

OROIAN, M. A. Cinética da temperatura de hidratação da Lens culinaris. Jornal da Sociedade Saudita de Ciências Agrícolas, 16 (3), 250-256. 2017.

PAIXÃO, A. A., CORRÊA, P. C., MACHADO, F. B., LOPES, S. M. D., DINIZ, M. D. M. S., FREITAS, R. L. Modeling the hydration process of bean grains coated with carnauba wax. Semina: Ciências Agrárias, 38(4). 2017.

SINGH, R. K., MAJUMDAR, R. K., VENKATESHWARLU, G. Effect of Process Conditions on Physico- Chemical and Sensory Properties of Fish- Cereal- Based Extruded SnackLike Products. Journal of Food Processing and Preservation, v. 38, n. 1, p. 68-82. 2014.

VOLPE, T., SANTOS, A., JORGE, L., JORGE, R. Estudo da cinética de hidratação e propriedades do grão de arroz durante o processo de parbolização. Blucher Chemical Engineering Proceedings, 1(2), 2950-2957. 2015. 
